\title{
A Mobile Application for Physical Activity Recognition using Acceleration Data from Wearable Sensors for Cardiac Rehabilitation
}

\author{
M. Chaari ${ }^{1,2}$, M. Abid ${ }^{2,3}$, Y. Ouakrim ${ }^{2,3}$, M. Lahami ${ }^{1}$ and N. Mezghani ${ }^{2,3}$ \\ ${ }^{1}$ National School of Engineers of Sfax, Sfax University, Tunisia \\ ${ }^{2}$ LICEF Research Center, TELUQ, Montreal, Canada \\ ${ }^{3}$ Laboratoire de Recherche en Imagerie et Orthopédie (LIO), CRCHUM, Montreal, Canada
}

\begin{abstract}
Keywords: mHealth, Mobile Application, Cardiac Rehabilitation, Human Activity Recognition (HAR), Wearable Sensors, Classification.

Abstract: $\quad$ mHealth applications are an ever-expanding frontier in today's use of technology. They allow a user to record health data and contact their doctor from the convenience of a smartphone. This paper presents a first version release of a mobile application that aims to assess compliance of cardiovascular diseased patients with home-based cardiac rehabilitation, by monitoring physical activities using wearable sensors. The application generates reports for both the patient and the doctor through an interactive dashboard, as initial proposal, that provides feedback of physical activities of daily living undertaken by the patient. The application integrates a human activity recognition system, which learns a support vector machine algorithm to identify 10 different daily activities, such as walking, going upstairs, sitting and lying, from accelerometer data using a connected textile including movement sensors. Our early deployment and execution results are promising since they are showing good accuracy for recognizing all the ten daily living activities.
\end{abstract}

\section{INTRODUCTION}

Cardiac rehabilitation (CR) is a systematic model of chronic vascular disease care that pro-actively monitors these conditions using a multi-faceted approach. This approach includes behavior change strategies related to a sustainable lifestyle and adherence to pharmacological treatment as well as therapeutic exercises and physical activity programs to improve secondary prevention outcomes in patients with cardiovascular disease or recovering from surgery. CR reduces total mortality and cardiac mortality by 20 to $25 \%$ (Cyr et al., 2018). It may also reduce the number of hospitalizations related to heart disease and the need for new revascularization procedures in patients with coronary artery disease. However, only a minority of eligible patients participate in CR programs. Homebased cardiac rehabilitation (HBCR) is definitely one of the new urgently needed strategies to improve the participation rate. It uses remote coaching with indirect exercise supervision and helps limit hospital or clinic visits (Thomas et al., 2019). In recent decades, CR has evolved from simple surveillance aimed at a safe return to physical activity, to a multidisciplinary approach focused on patient education, personalized physical training, changing risk factors and the wellbeing of cardiac patients (Mampuya, 2012). More- over, recent advances in information and communication technologies have been used to enhance HBCR programs (Varnfield et al., 2011). Besides improving the quality of measures, wearable devices and portable medical sensors have also proven effective in monitoring a greater number of patients in prevention and rehabilitation programs in a personalized manner. As a result, and thanks to recent tools, the use of home-based mHealth programs has been increasing, achieving good control over vital signs and physical activities (Medina et al., 2017). Recent research studies in human activity recognition (HAR) have focused on sensor-based home monitoring systems. HAR is defined as the ability of an intelligent system to infer temporally contextualized knowledge regarding the state of the user and to classify a set of human activities on the basis of a set of sensor readings. Its role, extremely important in the burgeoning healthcare field, is to provide all the necessary data on the patient's health and well-being outside a hospital setting. Technological advances have made HAR a rapidly growing area, thanks to the use of affordable mobile platforms such as smart phones and other personal tracking devices (Damasevicius et al., 2016), which, together with body-worn sensors, can solve the cardiac patient monitoring problem. There are countless examples of applications that use hu- 
man activity recognition to indicate the health status of humans. Fitbit, for example, offers smart watches and fitness bands to allow healthcare professionals to track individuals and monitor their activity trends over time (Paul et al., 2015). Tactio Health, Samsung Health and many more are able to track the number of steps, sleep patterns, passive periods, etc (Voicu et al., 2019). They are designed to facilitate monitoring patients efficiently so doctors can have better control of their chronic conditions. Although these apps recognize a very limited number of activities, they have excellent results, being extremely accurate in detecting the type of activity performed.

Our study aims to develop a mobile application for monitoring physical activities by using wearable sensors as part of a HBCR program which is designed for patients who are unable to attend the traditional facility based cardiac rehabilitation. The application's goal is to help clinicians perform remote follow-up on their patients. It displays their daily routine activities on a simple dashboard, providing doctors with the necessary information to check patient's health condition. So this allows patients to exercise in their own homes while being supervised by one of the clinicians in cardiac rehabilitation all done using the mobile application. Patients also have access to the application so they can also check their improvement.

This work will be presented as follows: Section 2 analyzes the methods used in this project. In Section 3 , the system architecture is explained, with specification of the technologies chosen for the application development. Section 4 focuses on presenting the main results, and, finally, the conclusion is given in Section 5.

\section{METHODS AND MATERIALS}

The work presented here was carried out as a joint academic-industry research project designed to measure patient adherence to HBCR programs. In the present study, a mobile application is developed for activity monitoring based on data acquired by the Hexoskin intelligent textile shirt ${ }^{1}$, developed by Carré Technologies (Montreal, Canada). The Hexoskin is an easily donned, comfortable stretch shirt that can be used in any ambient environment. Data acquisition by the Hexoskin is non-invasive and can be performed continuously without hampering the movements of the person wearning it. Hexoskin offers the only clinically validated system that allows precise ECG cardiac monitoring with lung function and ac-

\footnotetext{
${ }^{1}$ https://www.hexoskin.com/
}

tivity monitoring over the long-term (Banerjee et al., 2015). Its measurements were found to be reliable in many research studies. This system offers a recording capacity of over 42,000 data points of scientific information per minute. The sensors are placed away from the chest area so users can safely engage in contact sports (Cherif et al., 2018a). A cable running from the shirt is plugged into the companion device that is secured in a waist pocket (Figure 1).

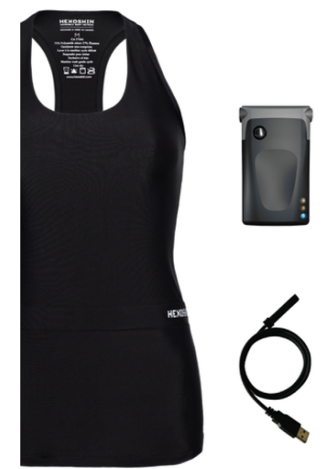

Figure 1: Hexoskin shirt, smart device and USB cable.

Once reception of body metrics data begins, the Hexoskin device can wirelessly stream the data to a mobile device in real-time, or store the information until the user is able to transfer it via the supplied USB cable. In practice, the Hexoskin enables realtime monitoring of cardiac, respiratory and 3D acceleration data via cardiac, breathing and movement embedded sensors, respectively (Figure 2). In this paper, we focus on movement sensors that calculate 3 -axis accelerations.

(a)

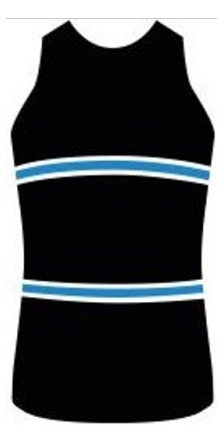

Figure 2: Hexoskin embedded sensors : (a) cardiac, (b) breathing and (c) movement sensors. The cardiac sensors allow to track the heart rate and display the electrocardiogram (ECG) in real time. The breathing sensors indicate the breathing capacity for a given activity. The movement sensors calculate 3 -axis accelerations, activity levels and steps.

This study extends previous research (Cherif et al., 2018b), by implementing a HAR framework on a 
mobile phone using 3D acceleration data. As illustrated in Figure 3, the HAR framework consists of two phases: the offline data training and the online classification.

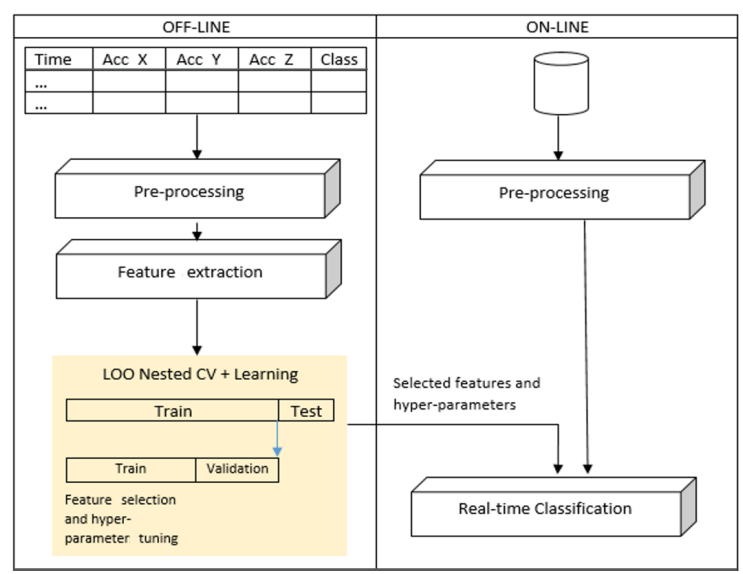

Figure 3: The HAR framework includes an offline learning and an online classification.

\subsection{Data Collection for Offline Learning}

The data collection has been approved by institutional ethics committees and all subjects provided written informed consent before participating. Data collection was performed at the research center of the University of Montreal Hospital Center (Montreal, Canada) and recruited participants were in good health and didn't have chronic pain or known locomotor or heart problems. Fourteen more healthy volunteers have been included in the pre-established data base with the same data collection protocol. A total of 40 voluntary participants wore the smart textile Hexoskin and performed 5 repeats of a sequence of 10 tasks: going up the stairs, going down the stairs, walking, running, sitting, falling right, falling left, falling backward, falling forward and lying down. The choice of these selected activities was made to represent the majority of everyday living activities (Attal et al., 2015). To ensure a good recording signal, patients were instructed to moisten the three gray electrodes inside the shirt, connect the Hexoskin device to the shirt connector and insert it horizontally into the shirt pocket with the wire upward and the light outward.

The accelerations were collected from the 3-axis sensors integrated into the Hexoskin. In total, the acceleration data of 40 healthy volunteers performing the same data collection protocol was collected and considered in the classification model. A video recording was made during the whole session. Its sequences were used later to label the different classes of physical activities.

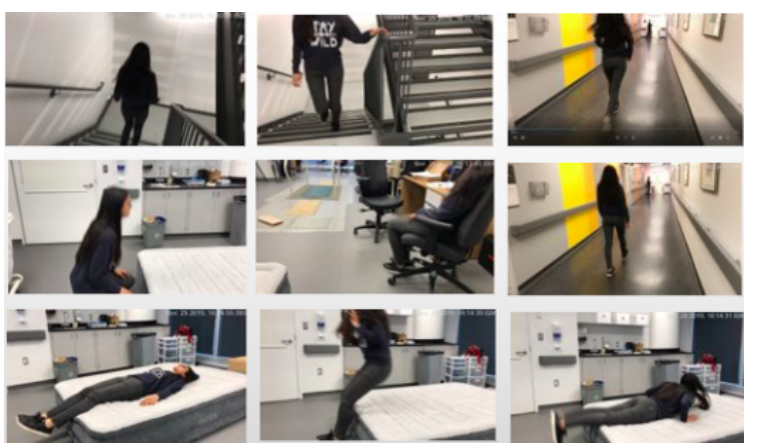

Figure 4: A scenario of data collection with a participant wearing the Hexoskin. Each sub-image corresponds to a task, i.e., an activity.

\subsection{Data Transmission for Online Classification}

When the shirt is unplugged, data recording ceases and the device automatically shuts down after $60 \mathrm{sec}-$ onds. The administrator logs in to the participant's Hexoskin account, connects the device to the computer with the USB cable and launches the HxServices application. The data start to synchronize automatically and are saved on the Hexoskin server.

In a real-life context, the patient would perform the data transmission daily using his own computer to transfer his records from the smart device to the Hexoskin server. Then, he can launch the mobile application. Once he does, a request is sent to the Hexoskin server via the Application Programming Interface (API) ${ }^{2}$ (Application Programming Interface) to get the patient's data. Upon receiving the request, the Hexoskin server sends a response.

\subsection{Classification}

The collected acceleration data are trained, during the offline phase, using a machine learning classification algorithm which returns the resulting activities in label form.

As shown in Figure 5, the machine learning classification algorithm consists of the following steps: First, the acceleration norm is computed from the three axis acceleration signal. The sequence of the acceleration vector norm is then segmented into short time sequences using a rectangular window around each detected peak. More precisely, peaks are detected by thresholding the norm. Time-domain and frequency domain features are computed on 1s-length windows from the $3 \mathrm{D}$ acceleration data. The Relief-F algorithm is then used to rank individual features ac-

\footnotetext{
${ }^{2}$ https://api.hexoskin.com/docs/page/oauth2/
} 
cording to their relevance scores. After the selection process, the top ten features were retained to be used for the classification. The next step is a physical activity classification system developed using Matlab. The classification uses the Support Vector Machine (SVM) classifier. It is a supervised machine learning algorithm that aims to find the optimal separating decision hyperplanes between classes with the maximum margin between patterns of each class. The tests were done according to the process of Leaveone-subject-out cross validation for the SVM model. Tests of its performance, evaluated in terms of correct classification rates, have demonstrated the reliability of the approach with an accuracy of $95.4 \%$.

There has been numerous studies to investigate different machine learning models for physical human activity recognition using wearable sensors for acceleration signals. In (Attal et al., 2015), the authors proposed a classification methodology to recognize, using acceleration data, different classes of daily living human activities, by comparing different machine learning techniques namely, k-Nearest Neighbor (kNN), SVM, Gaussian Mixture Models (GMM), and Random Forest (RF). In our paper, the choice of the feature extraction, selection and classification methods was made just for deploying the HAR framework on mobile devices. The next step, which is our concern, is the implementation of the mobile application, and the deployment of our own custom model for HAR. We'll be detailing this step in the next section.

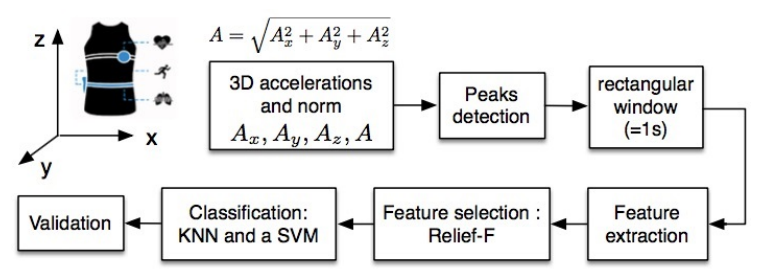

Figure 5: Block diagram of the proposed physical activity classification system as proposed in (Cherif et al., 2018b). In their work, the authors tested an SVM and a KNN classifier.

\section{APPLICATION ARCHITECTURE AND TECHNOLOGIES}

The objective of this project part was to develop a mobile application for physical activity classification from data collected by the Hexoskin textile. The application will be used to implement a tool to measure compliance with home rehabilitation treatments. The physiological classification and analysis systems as

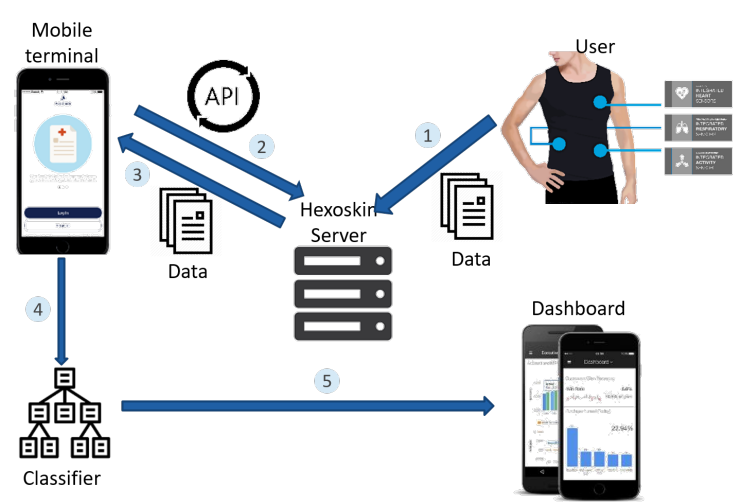

Figure 6: System scenario: data reception, HAR classification and local visualization. The Data is located in the Hexoskin server. The offline data training is performed in the backend server. Only the results are transplanted onto the mobile device dashboard.

well as the compliance measurement tool will be integrated in the future into the Hexoskin platform.

The collected data, analysis and patient local interface of the project was implemented in a smartphone application compatible with Android or iOS. This application as show in Figure 6 has the following main functions:

- Data reception (steps 1, 2 and 3): After data transmission from the Hexoskin shirt to the server, the application has to receive Hexoskin information packages from the server using an API. Then, the system has to extract the acceleration, cardiac and respiratory data.

- HAR classifier (step 4): The app uses a classifier algorithm to build a recognition model to estimate the current activity.

- Local visualization (step 5): All the latest sensor data can be seen in the mobile screen, in conjunction with the latest recognized activity.

Figure 7 presents the different parts of our application. The frontend comprises the presentation layer based on an Ionic 4 (Dunka et al., 2017), Angular $7^{3}$ and Cordova ${ }^{4}$ framework to produce a multiplatform application imitating the native behavior, grouping the Man-Machine interfaces and scripts that communicate with the backend based on the protocol Hypertext Transfer Protocol (HTTP) and Java Script Object Notation (JSON) exchange format.

The backend, corresponding to the second part, comprises the application server developed using Python $^{5}$ technology, since the classification model

\footnotetext{
${ }^{3}$ https://angular.io/

${ }^{4}$ https://cordova.apache.org/

${ }^{5} \mathrm{https} / / /$ www.python.org/
} 


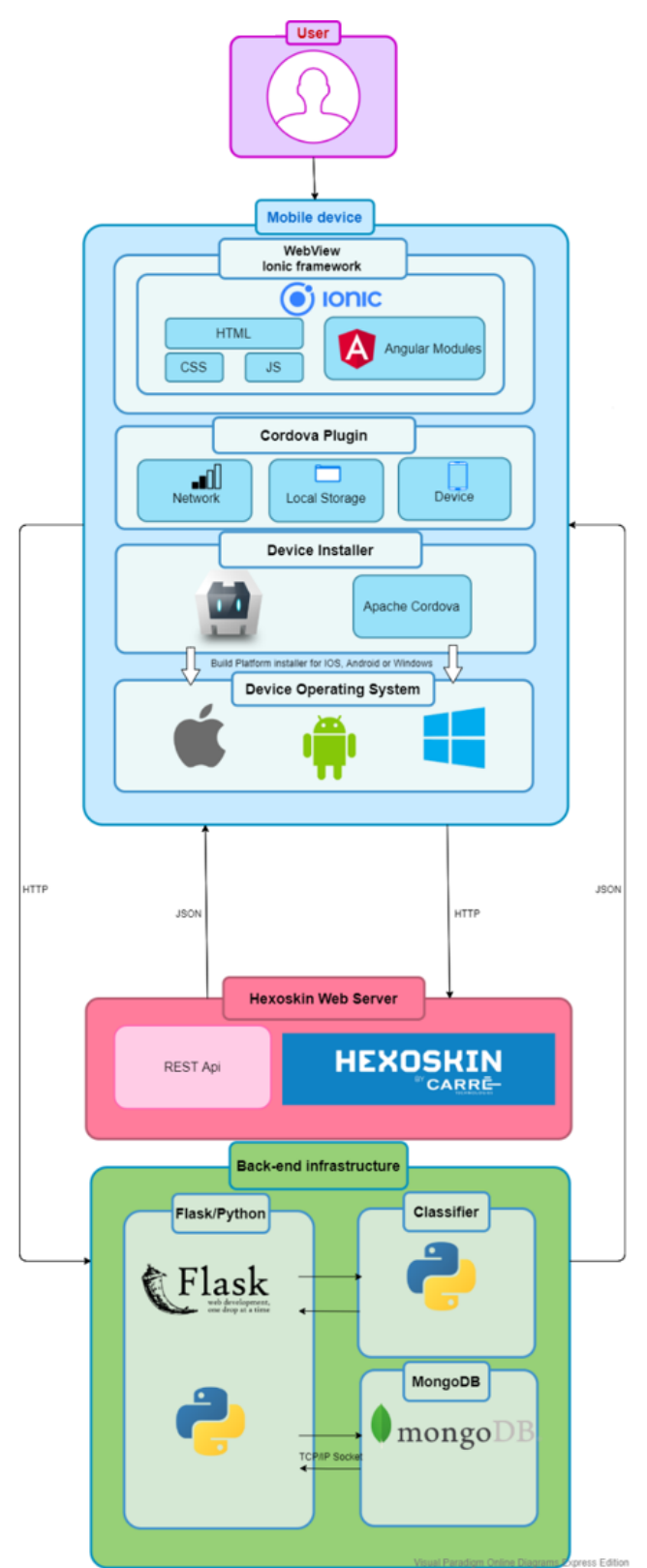

Figure 7: Global architecture of the application.

was converted from Matlab to Python in order to facilitate the connection with the front end. We used the Flask $^{6}$ framework provided with a lightweight Web server, which requires minimal configuration, can be controlled via Python code and can easily exchange information with MongoDB ${ }^{7}$, in which the data is stored.

The third part, the Hexoskin Web server (Webster et al., 2017), contains the data for the patient wearing

\footnotetext{
${ }^{6}$ http://flask.palletsprojects.com/en/1.1.x/

${ }^{7}$ https://www.mongodb.com/fr
}

the connected textile, and it is the Hexoskin Rest $\mathrm{API}^{8}$ and the classification system that make it possible to interact and manipulate the Hexoskin data via HTTP requests. This includes access to biometrics and user information, but also data annotation, advanced report visualization, metrics retrieval, and more.

\section{RESULTS}

This mobile application is developed for an mHealth context, that may be used for communication between a patient undertaken a HBCR and a clinician. However, we will only present screenshots and functionalities to illustrate the dashboard dedicated to the doctor. The patient's perspective will be considered in future work.

Authentication. Authentication is a process that performs identity verification. It is an important phase in order to secure the application. To access the various features of the application, the user must type his credential (login and password) into the corresponding fields (Figure 8).

Patient's Profile. The profile interface, as shown in Figure 8, is the first interface the user sees and interacts with. It provides a variety of patient information such as name, birth date, heart rate, weight, height,etc. The user can then access the main menu to discover the features of our application.

Dashboard. The dashboard depicted in Figure 9 shows the activities corresponding to the chosen record. Each activity is described by its name and duration. The patient's advancement is also recorded in the form of progress bars, allowing the doctor to check whether the patient is following the exercice program.

Medical Report Generation. After consulting the dashboard, the doctor can write his medical report. To do so, he clicks on "Write a report" to access the report form. He indicates his patient's state (improved, not improved or deteriorated), and then writes his notes concerning the records he has seen.

Medical Reports List. Each report is saved in the list (Figure 8) where it is indexed by date, content, doctor's name, record id and duration.

\footnotetext{
${ }^{8}$ https://api.hexoskin.com/docs/index.html\#api-keysand-oauth-requests
} 


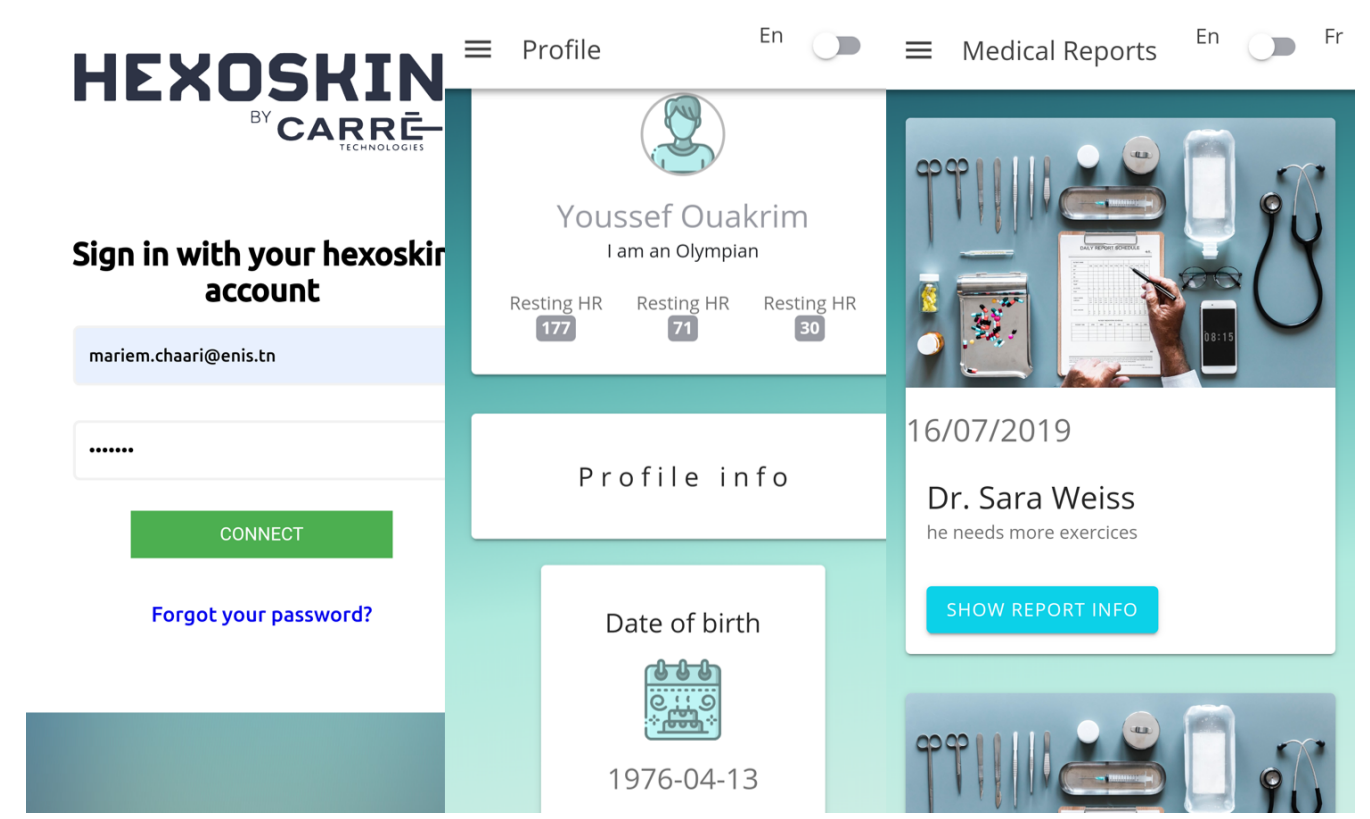

Figure 8: Login, patients profile and medical reports pages.

Patient's Program. The doctor can set out an exercice program for his patients, setting the performance durations of the individual activities as goals to reach.

This mHealth solution gathers information from the wearable sensors incorporated in an intelligent textile and process it using a HAR approach accessible via a mobile application. These kinds of applications provide a novel approach to the health care system, resulting in new and better services such as constant monitoring of patients and remote consultation services. Together, these services yield a faster and more reliable health care industry with almost zero waiting time, changing the classical approach of the health care system as a reactive service to that of a preemptive industry. In this project, the main focus is to improve the health care services for patients who, while their health status is not critical, still need constant monitoring. We have used the Hexoskin as an easy-to-wear washable textile that tracks heart rate, breathing rate and acceleration and has been used in several research studies showing its efficiency (Villar et al., 2015). Moreover, having all the data recorded in the server allows it to be accessed rapidly whenever needed, which is profitable in terms of energy and time. The classification model has an overall accuracy of $95.4 \%$ and a very short response time. In addition, we have developed an interactive interface which allows the doctor to monitor his patient's health easily from home.

The proposed HAR system is used for training and recognition of human activities in daily living, and in- tegrated in a mobile device as a first release version. An extensive work will be accomplished for training and recognition of physical excercices in rehabilitation process. The key requirements for the HAR system designed to support the rehabilitation process will be specified in conjunction with the rehabilitation team.

\section{CONCLUSIONS}

In this paper, we have presented a multi-platform HAR-based mobile application for HBCR. We developed an application that allows doctors to monitor targeted patients at their homes, using the Hexoskin intelligent-textile shirt. The shirt records acceleration signals which are then sent to our application for analysis and classification, in order to recognize one of the following activities: walking, running, falling right, falling left, falling backward, falling forward, going up stairs, going down stairs, sitting and lying. The application allows the doctor to visualize the textile recordings, each representing wearing of the Hexoskin for one day, and to write reports about these records. The idea was to design an archive of medical reports as a reference the doctor can use to better judge a patient's condition. We aim to increase the patients particpating rate in HBCR programs thanks to this system, so that they can lead a normal and safe life without having to worry about their health. The work process involved several stages in order to accomplish the development and implementation of the 


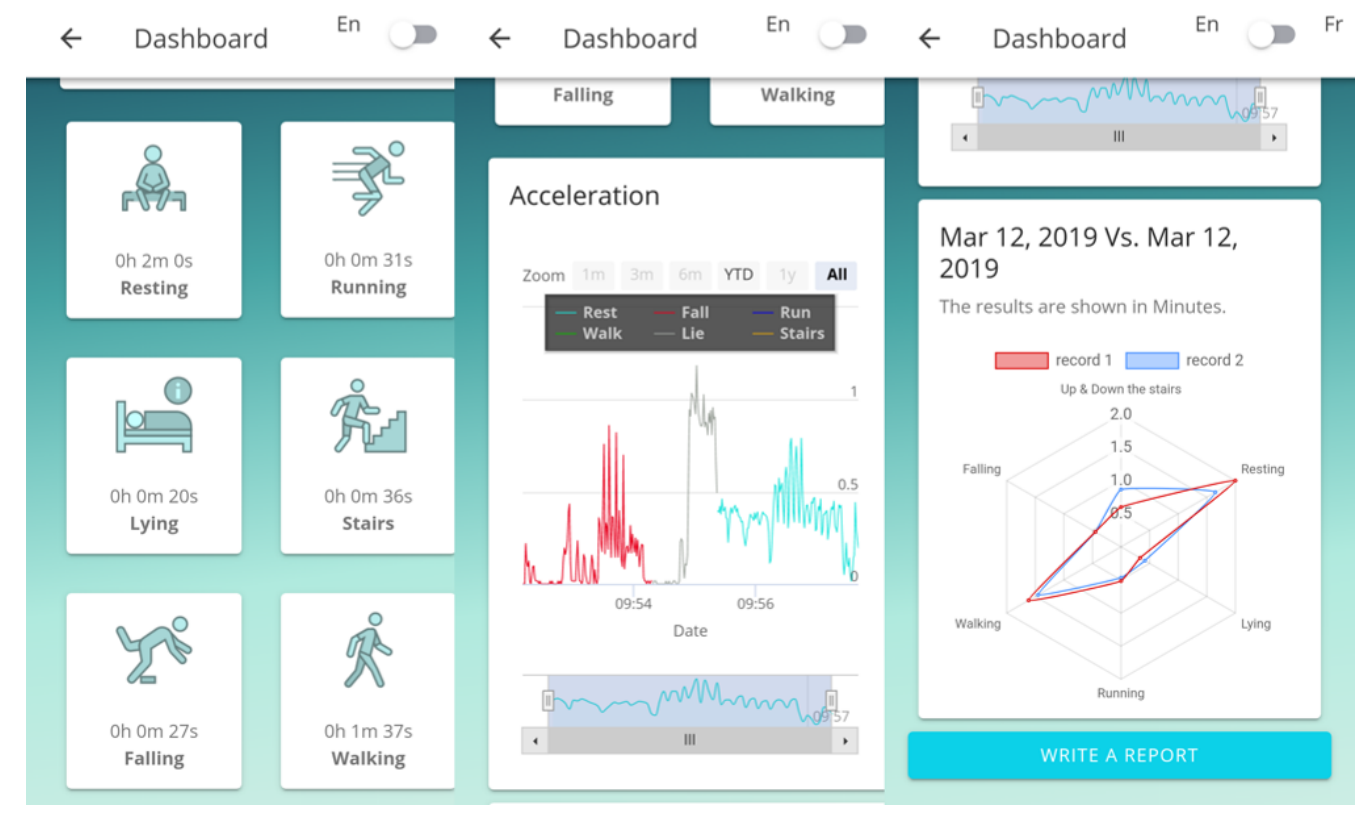

Figure 9: The dashboard page.

application and to collect the data. The collection of data for the new database was also an important phase in developing the classification system. However, this application is not limited to integrated functionalities such as monitoring patient's activity and writing reports.

In future work, we aim to add several types of exercises that are included in a cardiac rehabilitation program according to the guidelines of the American College of Sports Medicine (ACSM). In conjuction with a cardiac rehabilitation team, we will work on gathering empirical data from a set of patients with heart failure following a cardiac rehabilitation program. While, the activities we have examinated so far are quite simple, many of them could in fact be part of more complex routines or behaviors. For instance, swimming and cycling are composed of several instances of walking, running, and sitting (among other activities) characterized by a certain logical sequence and duration. Moreover, integrating cardiac and respiratory signals into the medical decision and the classification system would be important for a better classification rate. Future expanded datasets should also include more participants, especially patients in rehabilitation. We intend also to conduct an extensive set of experiments to compare different machine learning and deep learning approaches, especially ensemble methods, for human activity recognition using the acceleration data from the Hexoskin.

\section{REFERENCES}

Attal, F., Mohammed, S., Dedabrishvili, M., Chamroukhi, F., Oukhellou, L., and Amirat, Y. (2015). Physical Human Activity Recognition Using Wearable Sensors. Sensors, 15(12):31314-31338.

Banerjee, T., Anantharam, P., Romine, W., Lawhorne, L., and Sheth, A. (2015). Evaluating a potential commercial tool for healthcare application for people with dementia. In the International Conference on Health Informatics and Medical Systems.

Cherif, N., Mezghani, N., Gaudreault, N., Ouakrim, Y., Mouzoune, I., and Boulay, P. (2018a). Physiological data validation of the hexoskin smart textile. In BIODEVICES, pages 150-156.

Cherif, N., Ouakrim, Y., Benazza-Benyahia, A., and Mezghani, N. (2018b). Physical activity classification using a smart textile. In 2018 IEEE Life Sciences Conference ( $L S C$ ), pages 175-178.

Cyr, C., Robert, S., and Sylvie, P. (2018). Modèles de réadaptation cardiaque alternatifs utilisant les structures de gestion de cas et des maladies chroniques. 40:4-6.

Damasevicius, R., Vasiljevas, M., Salkevicius, J., and Woźniak, M. (2016). Human activity recognition in aal environments using random projections. Computational and Mathematical Methods in Medicine, 2016:1-17.

Dunka, B., Emmanuel, E., and Oyerinde, Y. (2017). Hybrid mobile application based on ionic framework technologies. International Journal of Recent Advances in Multidisciplinary Research, 04:3121-3130.

Mampuya, W. (2012). Cardiac rehabilitation past, present 
and future: An overview. Cardiovascular Diagnosis Therapeutics, 2:38.

Medina, J., Olmo, M., Aguilera, M., and Espinilla, M. (2017). Real-time monitoring in home-based cardiac rehabilitation using wrist-worn heart rate devices. Sensors, 17.

Paul, S. S., Tiedemann, A., Hassett, L. M., Ramsay, E., Kirkham, C., Chagpar, S., and Sherrington, C. (2015). Validity of the fitbit activity tracker for measuring steps in community-dwelling older adults. BMJ Open Sport \& Exercise Medicine, 1(1).

Thomas, R., Beatty, A., Beckie, T., Brewer, L., Brown, T., Forman, D., Franklin, B., Keteyian, S., Kitzman, D., Regensteiner, J., Sanderson, B., and Whooley, M. (2019). Home-based cardiac rehabilitation: A scientific statement from the american association of cardiovascular and pulmonary rehabilitation, the american heart association, and the american college of cardiology. Journal of Cardiopulmonary Rehabilitation and Prevention, 39:1.

Varnfield, M., Karunanithi, M., Särelä, A., Garcia, E., Fairfull, A., Oldenburg, B., and Walters, D. (2011). Uptake of a technology-assisted home-care cardiac rehabilitation program. The Medical journal of Australia, 194:15-19.

Villar, R., Beltrame, T., and Hughson, R. (2015). Validation of the hexoskin wearable vest during lying, sitting, standing and walking activities. Applied Physiology, Nutrition, and Metabolism, 40:1019-24.

Voicu, R.-A., Dobre, C., Bajenaru, L., and Ciobanu, R.I. (2019). Human physical activity recognition using smartphone sensors. Sensors, 19:458.

Webster, E., Sukaviriya, N., Chang, H.-Y., and Kozloski, J. (2017). Predicting cognitive states from wearable recordings of autonomic function. IBM Journal of Research and Development, 61:2:1-2:11. 